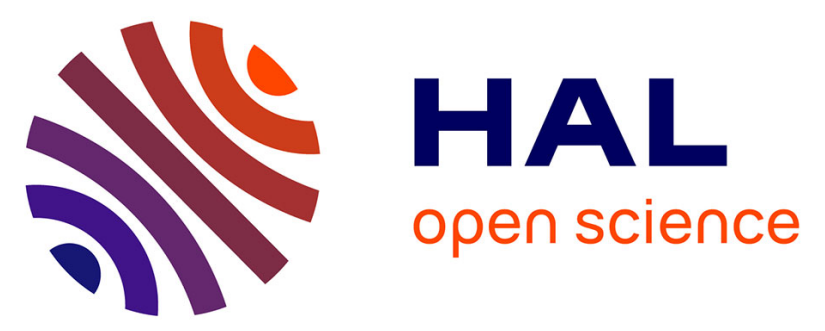

\title{
Paternal age negatively affects sperm production of the progeny
}

\author{
Pauline Vuarin, Loïc Lesobre, Gwènaëlle Levêque, Michel Saint Jalme, \\ Frédéric Lacroix, Yves Hingrat, Gabriele Sorci
}

\section{To cite this version:}

Pauline Vuarin, Loïc Lesobre, Gwènaëlle Levêque, Michel Saint Jalme, Frédéric Lacroix, et al.. Paternal age negatively affects sperm production of the progeny. Ecology Letters, 2021, 24 (4), pp.719-727. 10.1111/ELE.13696 . hal-03257302

\section{HAL Id: hal-03257302 https://hal.science/hal-03257302}

Submitted on 10 Jun 2021

HAL is a multi-disciplinary open access archive for the deposit and dissemination of scientific research documents, whether they are published or not. The documents may come from teaching and research institutions in France or abroad, or from public or private research centers.
L'archive ouverte pluridisciplinaire HAL, est destinée au dépôt et à la diffusion de documents scientifiques de niveau recherche, publiés ou non, émanant des établissements d'enseignement et de recherche français ou étrangers, des laboratoires publics ou privés. 
Pauline Vuarin ${ }^{1,2 \#}$, Loïc Lesobre ${ }^{1}$, Gwènaëlle Levêque ${ }^{3}$, Michel Saint Jalme ${ }^{4}$, Frédéric Lacroix ${ }^{1}$,

$4 \quad{ }^{1}$ Reneco International Wildlife Consultants LLC; Abu Dhabi, PoBox 61741, United Arab Emirates

52 Biogéosciences, UMR 6282 CNRS, Université de Bourgogne Franche-Comté, 6 boulevard Gabriel, 621000 Dijon, France

$7 \quad{ }^{3}$ Emirates Center for Wildlife Propagation, BP 47, route de Midelt, 33250 Missour, Morocco

$8{ }^{4}$ Centre d'Ecologie et des Sciences de la Conservation, UMR 7204 MNHN CNRS-UPMC, Museum

9 National d'Histoire Naturelle, 43 et 61 rue Buffon, 75005 Paris, France

\# Current address : Laboratoire de Biométrie et Biologie Evolutive - UMR CNRS 5558, Université Lyon

1, 16 rue Raphaël Dubois, 69622 Villeurbanne cedex, France

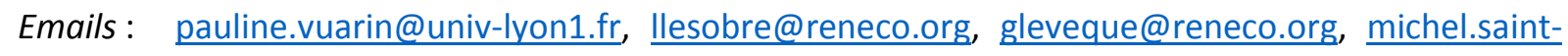

Running title : Paternal age and offspring sperm production

Article type : Letters

Key words: Aging, ejaculate quality, male gametes, offspring phenotype, paternal age, postcopulatory sexual selection, reproductive senescence

Word count: abstract (125), main text (4209).

References: 60

Tables: 2

Figures: 2

* Corresponding author: Gabriele Sorci, Biogéosciences, UMR 6282 CNRS, Université de Bourgogne 
25 Author contribution : PV, MSJ, LL, YH and GS conceived the study; LL, GL, FL, and YH supervised the 26 long-term data collection; PV and GS analysed the data and wrote the first draft of the manuscript;

27 PV, LL, GL, MSJ, FL, YH and GS revised the manuscript and approved its final version. Data availability statement: Data will be deposited in DRYAD upon acceptance of the manuscript.

29 


\section{Abstract}

Parental age has profound consequences for offspring's phenotype. However, whether patrilineal age affects offspring sperm production is unknown, despite the importance of sperm production for male reproductive success in species facing post-copulatory sexual selection. Using a longitudinal dataset on ejaculate attributes of the houbara bustard, we showed that offspring sired by old fathers had different age-dependent trajectories of sperm production compared to offspring sired by young fathers. Specifically, they produced less sperm (-48\%) in their first year of life, and $14 \%$ less during their lifetime. Paternal age had the strongest effect, with weak evidence for grandpaternal or great grandpaternal age effects. These results show that paternal age can affect offspring reproductive success by reducing sperm production, establishing an intergenerational link between aging and sexual selection.

政

.
. 45

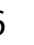
4 48 9 50 51

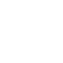
. 


\section{Introduction}

The idea that the parental phenotype can affect offspring's phenotype has been investigated in many species, and there is ample evidence showing that these "parental effects" have important consequences for offspring fitness and for the evolutionary dynamics of phenotypic traits (Mousseau and Fox 1998; Badyaev and Uller 2009; Crean and Bonduriansky 2014).

Age is among the parental traits that can affect offspring phenotype (Hercus and Hoffmann 2000; Kern et al. 2001; Priest et al. 2002; Carnes et al. 2012; Qazi et al. 2017; Gillespie et al. 2013; Lippens et al. 2017; Arslan et al. 2017). Reproductive senescence is a widespread phenomenon in nature (Nussey et al. 2013; Jones et al. 2014), and involves the sequential alteration of several functions from gamete production to parental care, ultimately resulting in an age-associated reduction in the number of offspring produced (Lemaître and Gaillard 2017). Furthermore, negative effects of aging can extend well beyond the number of offspring produced, since parental age can also impinge on the performance of the progeny, including traits that are expressed at early (e.g., embryo or juvenile survival, offspring development) and late life (e.g., lifetime reproductive success or longevity, the so-called Lansing effect) (Tarin et al. 2005; Garcia-Palomares et al. 2009; Lind et al. 2015; Bouwhuis et al. 2015; Schroeder et al. 2015; Fay et al. 2016).

Deleterious effects of parental age on offspring traits can be mediated by different mechanisms, involving environmental, epigenetic and genetic effects (Beamonte et al. 2010; Kong et al. 2012; Jenkins et al. 2014; Chen et al. 2016; Herati et al. 2017; Jónsson et al. 2017; Noguera et al. 2018; Bauch et al. 2019).

While few studies have investigated the effect of parental age on offspring lifespan and lifetime reproductive success (e.g., Bouwhuis et al. 2015; Schroeder et al. 2015), even less have focused on how parental age shapes the production of male gametes. In species where females mate with several males during the same reproductive bout, investment into sperm production is a key trait affecting male fitness (Parker and Pizzari 2010). Sperm competition and female cryptic choice have been shown to be strong selective forces shaping investment into ejaculate traits and sperm 
phenotype (Firman and Simmons 2009; Fitzpatrick and Lüpold 2014; Vuarin et al. 2019b). Therefore, if parental age impinges on traits involved in post-copulatory sexual selection, this is likely to have consequences for offspring reproductive success, establishing a link between aging and sexual selection (Bonduriansky et al. 2008). Despite its potential ecological and evolutionary relevance, to the best of our knowledge, the effect of parental age on male offspring reproductive function has only been investigated in humans (Sharma et al. 2015) and inbred strains of mice (Caballero-Campo et al. 2018; Xie et al. 2018). While findings generally point towards a negative effect of advanced parental age on male reproductive function, no study has investigated the persistence of these effects over more than two generations. However, such data might prove very valuable to indirectly infer the underlying mechanisms and the possible ecological and evolutionary consequences of such intergenerational effects. For instance, if parents reproducing at old age are more likely to transmit de novo mutations to their offspring, we might expect that deleterious effects of parental age persist across generations. Conversely, if parental effects arise because old parents provide different environmental conditions to their offspring, or due to epigenetic changes transmitted with gametes, we might expect strongest effects between parents and offspring (compared to grandparents/grandoffspring or great grandparents/great grandoffspring). However, exceptions to this expected pattern do exist, since epigenetic effects have been shown to persist across two generations (see for instance, Eisenberg et al. 2012), and deleterious age-associated mutations of the germline can be purged by negative selection.

Here, we took advantage of a longitudinal dataset that has been collected in a long-lived bird species, the North African houbara bustard (Chlamydotis undulata undulata), maintained in a conservation breeding program, to explore the effect of parental age on age-specific ejaculate traits of the progeny. Previous work conducted on this species has shown that number of sperm and an index of sperm motility improve with age and then decline as birds become older (Preston et al. 2011). Reproductive success of old males is also impaired, since eggs fertilized by senescing males have lower hatching success, hatchlings have reduced growth, and suffer higher post-release 
mortality (for female offspring) (Preston et al. 2015; Vuarin et al. 2019a). Moreover, post-copulatory sexual selection has been shown to operate on ejaculate traits in this species (Vuarin et al. 2019a,b). We first explored the effect of maternal and paternal age on offspring ejaculate traits. Since we found evidence for paternal age effects on sperm production, we subsequently extended the analysis including grandpaternal and great grandpaternal age, to investigate whether the effects persisted through patrilines.

\section{Materials and methods}

All birds used in this study are part of the Emirates Center for Wildlife Propagation (ECWP), a conservation breeding program located in eastern Morocco, aiming at reinforcing natural populations of the North African houbara bustard. This program relies entirely on artificial inseminations (Lesobre et al. 2010).

Ejaculates are routinely collected using a dummy female and immediately brought into an adjacent laboratory where the proportion of motile sperm, the number of sperm, the volume and the proportion of morphologically normal sperm in the ejaculate are assessed (see online material for details). Females are regularly checked for reproductive status and inseminated with freshly collected semen when ready to lay. Laid eggs are collected every morning and incubated following a standard protocol.

We investigated the potential effect of parental age on the following ejaculate traits: i) the mean number of sperm in the ejaculate per breeding season, ii) the mean mass motility index per breeding season, iii) the mean proportion of sperm with normal morphology in the ejaculate per breeding season. Data on age-specific sperm quantity and mass motility index were available for 1,708 individuals, from 1 to 17 years old, born from 2002 to 2018 and collected between 2003 and 2019. These 1,708 individuals were produced by 989 dams and 682 sires. The overall data set included 11,107 observations. Dam age ranged from 1 to 13 years (mean $\pm s d=3.78 \pm 1.85$ ), and sire age from 1 to 14 years (mean $\pm s d=3.88 \pm 1.90$ ). Owing to small sample size, individuals older than 
132 grouped in the same age class. The correlation coefficient between maternal and paternal age was 133 low $(r=0.095, n=1,708)$, showing that the two variables convey independent information on parental age. Data on the proportion of normal sperm were available for a smaller number of individuals (1,270 individuals produced by 758 dams and 543 sires, for a total of 2,273 observations). age at fertilization) was certain. This was ensured because females were either inseminated with the semen of a single male, or in case of multiple inseminations with different males, microsatellite markers were used to perform parentage analyses and identify the sire (Lesobre et al. 2010).

\section{Statistical analyses}

We used general additive models (GAM) to infer the shape of age-dependent variation of each of the three ejaculate attributes considered here. GAM is a nonparametric regression that relaxes the assumption of linearity and allows to explore traits that vary non-linearly without the constraints that are usually associated with polynomial models.

We used linear mixed models (LMM) to explore the effect of maternal and paternal age on age-dependent variation of ejaculate traits of the offspring. We fitted separate models for each response variable [i.e., number of sperm in the ejaculate (log-transformed), mass motility index, and the proportion of sperm with normal morphology]. All models had the same structure (normal distribution of errors), with the following terms fitted as fixed effects: offspring age (logtransformed), squared offspring age, maternal age, and paternal age. We also fitted the two-way interactions of parental age with offspring age and squared offspring age to test whether agedependent trajectories were affected by parental age. The random effects included offspring, maternal and paternal identities, year of birth and year of data collection. If individuals with particular phenotypic traits enter or exit from the sampled population at different ages (selective appearance and disappearance), this might generate a pattern of age-dependent variation at the 
population level, potentially leading to erroneous conclusions about the age-dependent trajectories at the individual level. To infer the within-individual variation with age, independently from the population-level (between-individual) variation caused by selective appearance and/or disappearance, we followed the methods advocated by van de Pol and Verhulst (2006), and included age at first insemination (that determines at which age a given individual enters the breeding population), and age at last insemination (that determines at which age a given individual exits the breeding population) as fixed effects. We started with the model that included all single effects and interactions and we sequentially dropped those that were not statistically significant, starting first with interactions and then with single effects, until obtaining a minimal adequate model. Note that age at first and last insemination were always kept in the models.

To investigate the effect of patrilineal age (over three generations) on sperm production, we used two approaches. First, we grouped birds according to the age of their fathers, grandfathers and great grandfathers. Ascendants that bred between 1 and 5 years were considered young, ascendants that bred when older than 5 years were considered old. Therefore, birds that consistently had young (y) ascendants were considered to have a "yyy" patrilineal age (the first letter referring to paternal age, the last letter to great grandpaternal age); birds that consistently had old (o) ascendants were considered to have a "ooo" patrilineal age. Two age classes and three generations give 8 possible patrilineal age groups (yyy, yyo, yoo, ooo, oyy, ooy, yoy, oyo). The effect of patrilineal age on sperm production was assessed running 6 LMMs where different patrilineal age groups were contrasted. Due to small sample size, we could not include ooy, oyo, ooy, ooo groups in the models. Each model had the same structure, with offspring age and squared age, patrilineal age (plus interactions), age at first and age at last insemination included as fixed effects. The random effects included offspring identity, maternal and paternal identity, year of birth and year of data collection. Restricting the sample size to individuals with known father, grandfather and great grandfather reduced the number of individuals included in the model to 888 , for a total of 5,504 observations. Second, we ran a LMM where the three ascendant ages were included in the same model, as continuous variables. This 
model therefore relaxed the constraint associated with the cutoff used to categorize birds as young or old. The model included offspring identity, maternal and paternal identity, year of birth and year of data collection as random effects.

GAMs and LMMs were performed using PROC GAM and PROC MIXED (SAS, 14.3), respectively.

\section{Results}

The GAMs showed that both the number of sperm in the ejaculate (i.e., sperm production), and the mass motility index increased up to the age of 5 years and then gradually declined as individuals became older, with a strong quadratic pattern (number of sperm in the ejaculate: $\chi^{2}=$ 582.04, $d f=3, n=11,107 ; p<0.0001 ;$ mass motility index: $\chi^{2}=413.47, d f=3, n=11,107, p<0.0001$; fig. S1A,B). The proportion of morphologically normal sperm showed a more complex pattern of variation with age, suggesting a higher-order behavior and a later peak value $\left(7\right.$ years, $\chi^{2}=22.87, \mathrm{df}=$ $3, n=2,273, p<0.0001 ;$ fig. S1C).

The LMM performed on the number of sperm in the ejaculate showed that offspring agedependent trajectory was modulated by paternal age, as indicated by the highly significant interactions between paternal and offspring age (both linear and quadratic components, table 1). Maternal age was not a significant predictor of sperm production, nor of its age-dependent variation (table 1).

Grouping paternal age into two age classes [up to the peak age (i.e., from 1 to 5 years) and post-peak age (i.e., older than 5 years)], allowed to visualize the effect of sire age on the trajectories of age-dependent offspring sperm production (fig. 1; table S1). Offspring sired by old fathers had a lower sperm production (-16.9\% difference in LS-MEANS on back-transformed values) during their early life (1-5 years) compared to offspring sired by young fathers (LMM, paternal age: $t=-2.78, p=$ 0.0055, age at first insemination: $t=0.97, p=0.3299$; age at last insemination: $t=19.44, p<0.0001$; number of observations $=5,640$ ). The difference was very strong at the age of 1 , with offspring sired 
by old fathers showing a $47.9 \%$ reduction in their sperm production (LMM, paternal age: $t=-2.52, p$

$210=0.0122$, age at last insemination: $t=1.48, p=0.1388 ;$ number of observations $=401 ;$ fig. $2 A)$. During the post-peak age (over 5 years), offspring sired by old fathers still had a lower sperm production (7.6\%) compared to offspring sired by young fathers, but this difference was not statistically significant (LMM, paternal age: $t=-1.11, p=0.2651$, age at first insemination: $t=0.54, p=0.5907$; age at last insemination: $t=8.41, p<0.0001$; number of observations $=5,467)$. Nevertheless, over the entire lifespan, offspring sired by old fathers produced $14 \%$ less sperm per ejaculate than offspring sired by young fathers (LMM, paternal age: $t=-2.19, p=0.0283$, age at first insemination: $t$ $=2.64, p=0.0083 ;$ age at last insemination: $t=16.55, p<0.0001 ;$ number of observations $=11,107$; fig. 2B).

Given that sperm number and mass motility index positively covaried $(r=0.736, n=11,107)$, we ran an additional LMM where the latter variable was included as a covariate. This model therefore aimed to investigate whether the effect of paternal age on sperm number still holds when keeping mass motility index constant. The model provided similar results than the previous one (table S2), suggesting that the effect of parental age on sperm number did not arise because of the covariation between sperm number and motility.

We also found that paternal age affected the age-dependent variation in offspring mass motility index [significant paternal age $\mathrm{x}$ offspring age (and squared age); table S3]. However, this pattern was entirely driven by the covariation with the number of sperm in the ejaculate (table S4). As for number of sperm in the ejaculate, maternal age had no effect on mass motility index, nor on its age-dependent variation (table S3).

Neither paternal nor maternal age modulated the proportion of morphologically normal sperm in the ejaculate or its age-dependent trajectory (table S5). generations. We grouped birds according to the age of their father, grandfather and great grandfather and ran separate models contrasting different patrilineal ages (table 2). Overall, we 
found that paternal age had the strongest effect on sperm production. For instance, comparing sperm production of birds with young or old fathers, but young grandfathers and great grandfathers, showed a marked reduction in early production of sperm in birds sired by old fathers (table 2, fig. S2). Unfortunately, some of the patrilineal age groups had very few birds making impossible to run all possible comparisons. Nevertheless, comparing birds that only differed in the great grandpaternal age class (yyy vs. yyo), also showed a negative effect of great grandpaternal age on sperm production (table 2, fig. S3), suggesting a persisting effect of paternal age over generations. A seemingly contradictory result came from the comparison between birds with young ascendants over the three generations (yyy) and birds with old grandfathers and great grandfathers (yoo). Indeed, in this comparison, yoo birds had the highest sperm production (table 2). It should be noted however, that the number of birds in this patrilineal age group was particularly small, compared to the yyy group (34 vs. 519), and as such the result of this model should be taken with caution.

Running a LMM where paternal, grandpaternal and great grandpaternal age were included in the same model as continuous variables provided similar results to those reported above, with paternal age having the strongest effect on offspring sperm production (table S6).

\section{Discussion}

We found that paternal but not maternal age affected sperm production of the progeny, and that this effect was the strongest over the one-generation transmission. Other ejaculate traits (mass motility index and the proportion of sperm with a normal morphology) were not affected by parental age.

Although the effect of parental age on offspring phenotype has been studied in several systems, most of this previous work has focused on maternal age, because it was believed that mothers had higher control over offspring phenotype. This view is, however, rapidly changing, and evidence is accumulating i) showing that paternal age is associated with several offspring phenotypic traits (Garcia-Palomares et al. 2009; Fay et al. 2016; Arslan et al. 2017; Caballero-Campo et al. 2018; 
Wylde et al. 2019); ii) identifying the possible underlying mechanisms (Curley et al. 2011; Kong et al. 2012; Soubry et al. 2014; Xie et al. 2018).

Assessing male reproductive function is a hard task in free-ranging animals because this requires repeated samples of ejaculates. Recent work has shown that paternal age negatively impinges on lifetime reproductive success of male house sparrows (Passer domesticus) (Schroeder et al. 2015), suggesting that one or several components of the male reproductive function are impaired when individuals are sired by an old father. Our results suggest that reduced gamete production might be one of the physiological mechanisms underlying the negative effect of paternal age on male offspring lifetime reproductive success, and especially so in species where males face postcopulatory sexual selection through sperm competition. This might be particularly relevant when paternal age affects the production of gametes during the early life of the descendants, when the force of selection is at its highest value. Number of gametes transferred during the mating is an important driver of siring success and especially so in species where females mate with several males and ejaculates compete for egg fertilization (Parker and Pizzari 2010). In the houbara bustard, we have previously shown that ejaculate attributes, such as motility, improve male siring success during competitive fertilizations (when sperm from different males compete within the female reproductive tract) (Vuarin et al. 2019b). Similarly, when females are inseminated with ejaculates of two males, the probability of siring success of the last male (last male precedence) decreases as the number of sperm of the first male used to inseminate the female increases (L. Lesobre, unpublished results). Therefore, the finding that paternal age impinges on sperm production has potentially far reaching ecological and evolutionary consequences. At the ecological level, parental age effects can be a phenotypic source of variation contributing to shape offspring quality and life history traits, with possible consequences in terms of population dynamics. In agreement with this hypothesis, experimental work conducted on soil mites (Sancassania berlesei) has shown that changes in life history traits (longer developmental time and larger size at maturity) due to maternal age lead to different population dynamics that persist over generations (Benton et al. 2008). At the evolutionary 
level, negative parental age effects on the progeny contribute to the age-associated decline of fitness and increase the strength of selection for earlier reproduction (Priest et al. 2002). Paternal age effects on the progeny might also alter the shape of the trade-off between early and late trait expression. One of the best supported predictions of the theory on the evolution of senescence is that high investment into early expressed traits comes at the price of reduced investment in late life, resulting in a negative correlation between early and late traits (Lemaître et al. 2015). However, offspring produced by old parents might have compromised quality and have impaired expression of fitness related traits over their entire reproductive life, flattening the relationship between early and late life traits. Finally, the extent of the costs associated with mating with an old partner also suggests that selection might have promoted mechanisms of avoidance of senescing males. In agreement with this hypothesis, we recently showed that female houbara bustards concomitantly inseminated with sperm from males at prime and post-prime age had a higher proportion of their eggs fertilized by prime age males (Vuarin et al., 2019a). This finding, therefore, suggests that postcopulatory selection might allow them to avoid the cost of being fertilized by senescing males. Whether such paternal age effects persist over generations remains an open question. We only found a moderate evidence for a negative effect of great grandparental age on sperm production, and the comparison between birds produced by consistently young ascendants and birds produced by young fathers and old grandfathers and great grandfathers, showed the opposite pattern (higher sperm production for the latter group). These results should be interpreted with caution, due to the heterogeneity in sample size (number of birds) in each patrilineal age group. age effects might arise because of age-associated changes in ejaculate attributes (Sharma et al. 2015). These effects have been named ejaculate-mediated paternal effects and can involve purely environmental, epigenetic or genetic mechanisms (see Immler 2018 and Evans et al. 2019 for recent reviews). During aging, both the cellular and non-cellular fraction of the ejaculate undergo profound changes that have the potential to further affect offspring phenotype. Sperm are particularly 

contained in seminal plasma play a crucial role for the preservation of sperm DNA integrity (Wai-sum et al. 2006) and during aging some of this protective function might be lost. Epigenetic changes can also occur in male germline cells (Xavier et al. 2019). DNA methylation in sperm is associated with advanced male age (Jenkins et al. 2014), can be transmitted to the progeny and affect offspring phenotype (Xie et al. 2018). However, epigenetic reprogramming can reverse methylation patterns (Law and Jacobsen 2010; Rando and Chang 2012; Zhang et al. 2020), resetting the system and explaining why epigenetic effects can be lost across generations. Other epigenetic factors such as histone modification, noncoding RNAs contained in the seminal plasma, or inheritance of shortened telomeres can also be at play (Chen et al. 2016; Immler 2018; Bauch et al. 2019). Finally, male germline is particularly prone to accumulate de novo mutations due to the process of cell division that occurs throughout the entire reproductive life of males. In agreement with this hypothesis, paternal age and the length of male reproductive life are good predictors of the number of de novo mutations passed on to the progeny in primates (Thomas et al. 2018); and inheritance of ageassociated de novo mutations has been identified as a possible risk factor for several offspring pathologies (e.g., Kong et al. 2012). In addition to the sperm nuclear contribution to the offspring, abnormalities in sperm centrioles can also affect offspring phenotype, although evidence has mostly been reported in humans (Avidor-Reiss et al. 2019).

Our experimental design allows us to discard several other possible causes of the negative parental age effects on sperm production. A common confounding factor in studies of parental age effects derives from the possible differential investment of females according to the perceived phenotypic quality of mates (Sheldon 2000). If old partners are perceived as poor mates, females might adaptively adjust their investment into eggs or offspring care, producing an apparent association between father age and offspring phenotype. However, reproduction of houbara 337 bustards kept in the captive breeding program is entirely based on artificial inseminations which implies that i) the paternal contribution is restricted to the ejaculate; ii) females are never in contact 
with males and therefore cannot adjust their investment into eggs according to male phenotype; iii) eggs are collected as soon as they are laid and thus there is no maternal care that might subsequently affect offspring quality. This last point might explain why we did not find any evidence for maternal age effects, if such effects occur through differential environmental conditions experienced by offspring of old vs. young mothers (Beamonte-Barrientos et al. 2010; Plaistow et al. 2015).

To conclude, we showed that paternal but not maternal age reduced sperm production in offspring. These delayed paternal effects have the potential to affect the strength of selection operating on post-copulatory traits and male reproductive fitness in the wild, establishing a link between ageing and sexual selection.

\section{Acknowledgments}

This study was funded by the Emirates Center for Wildlife Propagation (ECWP), a project of the International Fund for Houbara Conservation (IFHC). We are grateful to H.H. Sheikh Mohammed bin Zayed Al Nahyan, Crown Prince of Abu Dhabi and Chairman of the International Fund for Houbara Conservation (IFHC) and H.E. Mohammed Al Bowardi, Deputy Chairman of IFHC, for their support. This study was conducted under the guidance of Reneco International Wildlife Consultants LLC., a consulting company managing ECWP. We are thankful to all Reneco staff who helped collecting the data.

\section{Competing interest statement}

The authors declare no competing interest.

\section{References}

Aitken, R.J. (2020). Impact of oxidative stress on male and female germ cells: implication for fertility. Reproduction 159, R189-R201. 
Arslan, R.C., Willführ, K.P., Frans, E.M., Verweij, K.J.H., Bürkner, P.-C., Myrskylä, M. et al. (2017). Older fathers' children have lower evolutionary fitness across four centuries and in four populations. Proc. R. Soc. B 284, 20171562.

Avidor-Reiss, T., Mazur, M., Fishman, E.L. \& Sindhwani, P. (2019). The role of sperm centrioles in human reproduction - The known and the unknown. Front. Cell. Dev. Biol. 7, 188.

Badyaev, A.V. \& Uller, T. (2009). Parental effects in ecology and evolution: mechanisms, processes and implications. Phil. Trans. R. Soc. B 364, 1169-1177.

Bauch, C., Boonekamp, J.J., Korsten, P., Mulder, E. \& Verhulst, S. (2019). Epigenetic inheritance of telomere length in wild birds. PLoS Genet. 15, 1-15.

Beamonte-Barrientos, R., Velando, A., Drummond, H. \& Torres, R. (2010). Senescence of maternal effects: Aging influences egg quality and rearing capacities of a long-lived bird. Am. Nat. 175, 469-480.

Benton, T.G., St Clair, J.J.H. \& Plaistow, S.J. (2008). Maternal effects mediated by maternal age: From life histories to population dynamics. J. Anim. Ecol. 77, 1038-1046.

Bonduriansky, R., Maklakov, A., Zajitschek, F. \& Brooks, R. (2008). Sexual selection, sexual conflict and the evolution of ageing and life span. Funct. Ecol. 22, 443-453.

Bouwhuis, S., Vedder, O. \& Becker, P.H. (2015). Sex-specific pathways of parental age effects on offspring lifetime reproductive success in a long-lived seabird. Evolution 69, 1760-1771.

Caballero-Campo, P., Lin, W., Simbulan, R., Liu, X., Feuer, S., Donjacour, A. et al. (2018). Advanced paternal age affects sperm count and anogenital distance in mouse offspring. Reprod. Sci. 25, $515-522$.

Carnes, B.A., Riesch, R. \& Schlupp, I. (2012). The delayed impact of parental age on offspring mortality in mice. J. Gerontol. A Biol. Sci. 67, 351-357.

Chen, Q., Yan, W. \& Duan, E. (2016). Epigenetic inheritance of acquired traits through sperm RNAs and sperm RNA modifications. Nat. Rev. Genet. 17, 733-743. 
Cooper, E.B. \& Kruuk, L.E.B. (2018). Ageing with a silver-spoon: A meta-analysis of the effect of developmental environment on senescence. Evol. Lett. 2, 460-471.

Crean, A. \& Bonduriansky, R. (2014). What is a paternal effect? Trends Ecol. Evol. 29, 554-559.

Curley, J.P., Mashoodh, R. \& Champagne, F.A. (2011). Epigenetics and the origins of paternal effects. Horm. Behav. 59, 306-14.

Eisenberg, D.T.A., Hayes, M.G., Kuzawa, C.W. (2012). Delayed paternal age of reproduction $\mathrm{n}$ humans is associated with longer telomeres across two generations of descendants. Proc. Natl. Acad. Sci. USA 109, 10251-10256.

Evans, J.P., Wilson, A.J., Pilastro, A., Garcia-Gonzalez, F. (2019). Ejaculate-mediated paternal effects: evidence, mechanisms and evolutionary implications. Reproduction 157, R109-R126.

Fay, R., Barbraud, C., Delord, K. \& Weimerskirch, H. (2016). Paternal but not maternal age influences early-life performance of offspring in a long-lived seabird. Proc. R. Soc. B 283, 20152318.

Firman, R.C. \& Simmons, L.W. (2009). Experimental evolution of sperm quality via postcopulatory sexual selection in house mice. Evolution 64, 1245-1256.

Fitzpatrick, J.L. \& Lüpold, S. (2014). Sexual selection and the evolution of sperm quality. Mol. Hum. Reprod. 20, 1180-1189.

García-Palomares, S., Navarro, S., Pertusa, J.F., Hermenegildo, C., Garcia-Perez, M.A., Rausell, F. et al. (2009). Delayed fatherhood in mice decreases reproductive fitness and longevity of offspring. Biol. Reprod. 80, 343-349.

Gillespie, D.O.S., Russell, A.F. \& Lummaa, V. (2013). The effect of maternal age and reproductive history on offspring survival and lifetime reproduction in preindustrial humans. Evolution 67, 1964-1974.

Herati, A.S., Zhelyazkova, B.H., Butler, P.R. \& Lamb, D.J. (2017). Age-related alterations in the genetics and genomics of the male germ line. Fertil. Steril. 107, 319-323.

Hercus, M.J. \& Hoffmann, A.A. (2000). Maternal and grandmaternal age influence offspring fitness in Drosophila. Proc. R. Soc. B 267, 2105-2110. 
Immler, S. (2018). The sperm factor: paternal impact beyond genes. Heredity 121, 239-247.

Jenkins, T.G., Aston, K.I., Pflueger, C., Cairns, B.R. \& Carrell, D.T. (2014). Age-associated sperm DNA methylation alterations: possible implications in offspring disease susceptibility. PLoS Genet. 10, e1004458.

Jones, O.R., Scheuerlein, A., Salguero-Gomez, R., Camarda, C.G., Schaible, R., Casper, B.B. et al. (2014). Diversity of ageing across the tree of life. Nature 505, 169-173.

Jónsson, H., Sulem, P., Kehr, B., Kristmundsdottir, S., Zink, F., Hjartarson, E. et al. (2017). Parental influence on human germline de novo mutations in 1,548 trios from Iceland. Nature 549, $519-522$.

Kern, S., Ackermann, M., Stearns, S.C. \& Kawecki, T.J. (2001). Decline in offspring viability as a manifestation of aging in Drosophila melanogaster. Evolution 55, 1822-1831.

Kong, A., Frigge, M.L., Masson, G., Besenbacher, S., Sulem, P., Magnusson, G. et al. (2012). Rate of de novo mutations and the importance of father's age to disease risk. Nature 488:471-475.

Law, J.A. \& Jacobsen, S.E. (2010). Establishing, maintaining and modifying DNA methylation patterns in plants and animals. Nat. Rev. Genet. 11, 204-220.

Lemaître, J.-F., Berger, V., Bonenfant, C., Douhard, M., Gamelon, M., Plard, F. et al. (2015). Early-late life trade-offs and the evolution of ageing in the wild. Proc. R. Soc. B 282, 20150209.

Lemaître, J.-F. \& Gaillard, J.M. (2017). Reproductive senescence: new perspectives in the wild. Biol. Rev. 92, 2182-2199.

Lesobre, L., Lacroix, F., Caizergues, A., Hingrat, Y., Chalah, T. \& Saint Jalme, M. (2010). Conservation genetics of houbara bustard (Chlamydotis undulata undulata): population structure and its implications for the reinforcement of wild populations. Conserv. Genet. 11, 1489-1497.

Lind, M.I., Berg, E.C., Alavioon, G. \& Maklakov, A.A. (2015). Evolution of differential maternal age effects on male and female offspring development and longevity. Funct. Ecol. 29, 104-110.

Lippens, C., Faivre, B., Lechenault, C. \& Sorci, G. (2017). Aging parasites produce offspring with poor fitness prospects. Biol. Lett. 13, 20160888. 
Mousseau, T. \& Fox, C.W. (1998). Maternal effects as adaptations. New York: Oxford University Press.

Noguera, J.C., Metcalfe, N.B. \& Monaghan, P. (2018). Experimental demonstration that offspring fathered by old males have shorter telomeres and reduced lifespans. Proc. R. Soc. B 285, 20180268.

Nussey, D.H., Froy, H., Lemaitre, J.-F., Gaillard, J. \& Austad, S.N. (2013). Senescence in natural populations of animals: widespread evidence and its implications for bio-gerontology. Ageing Res. Rev. 12:214-225.

Parker, G.A. \& Pizzari, T. (2010). Sperm competition and ejaculate economics. Biol. Rev. 85, 897-934.

Plaistow, S.J., Shirley, C., Collin, H., Cornell, S.J. \& Harney, E.D. (2015). Offspring provisioning explains clone-specific maternal age effects on life history and life span in the water flea, Daphnia pulex. Am Nat. 186, 376-389.

Preston, B.T., Saint Jalme, M., Hingrat, Y., Lacroix, F. \& Sorci, G. (2011). Sexually extravagant males age more rapidly. Ecol. Lett. 14, 1017-1024.

Preston, B.T., Saint Jalme, M., Hingrat, Y., Lacroix, F. \& Sorci, G. (2015). The sperm of aging male bustards retards their offspring's development. Nat. Commun. 6, 6146.

Priest, N.K., Mackowiak, B., Promislow, D.E.L., May, N. \& Low, E.L.P.I.S. (2002). The role of parental age effects on the evolution of aging. Evolution 56, 927-935.

Qazi, M.C.B., Miller, P.B., Poeschel, P.M., Phan, M.H., Thayer, J.L. \& Medrano, C.L. (2017). Transgenerational effects of maternal and grandmaternal age on offspring viability and performance in Drosophila melanogaster. J. Insect. Physiol. 100, 43-52.

Rando, T.A. \& Chang, H.Y. (2012). Aging, rejuvenation, and epigenetic reprogramming: resetting the aging clock. Cell 148, 46-57.

Schroeder, J., Nakagawa, S., Rees, M., Mannarelli, M.-E. \& Burke, T. (2015). Reduced fitness in progeny from old parents in a natural population. Proc. Natl. Acad. Sci. USA 112, 4021-4025. 
Sharma, R., Agarwal, A., Rohra, V.K., Assidi, M., Abu-Elmagd, M. \& Turki, R.F. (2015). Effects of increased paternal age on sperm quality, reproductive outcome and associated epigenetic risks to offspring. Reprod. Biol. Endocrinol. 13, 1-20.

Sheldon, B.C. (2000). Differential allocation: tests, mechanisms and implications. Trends Ecol. Evol. 15, 397-402.

Soubry, A., Hoyo, C., Jirtle, R.L., Murphy, S.K. (2014). A paternal environmental legacy: Evidence for epigenetic inheritance through the male germ line. BioEssays 36, 359-371.

Tarín, J.J., Gomez-Piquer, V., Rausell, F., Navarro, S., Hermenegildo, C. \& Cano, A. (2005). Delayed motherhood decreases life expectancy of mouse offspring. Biol. Reprod. 72, 1336-1343.

Thomas, G.W.C., Wang, R.J., Puri, A., Harris, R.A., Raveendran, M., Hughes, D.S.T. et al. (2018). Reproductive longevity predicts mutation rates in primates. Curr. Biol. 28, 3193-3197.

van de Pol, M., Verhulst, S. (2006). Age-dependent traits: a new statistical model to separate withinand between-individual effects. Am. Nat. 167, 766-773.

Vuarin, P., Bouchard, A., Lesobre, L., Leveque, G., Chalah, T., Saint Jalme, M. et al. (2019a). Postcopulatory sexual selection allows females to alleviate the fitness costs incurred when mating with senescing males. Proc. R. Soc. B 286, 20191675.

Vuarin, P., Hingrat, Y., Lesobre, L., Saint Jalme, M., Lacroix, F. \& Sorci G. (2019b). Sperm competition accentuates selection on ejaculate attributes. Biol. Lett. 15, 20180889.

Wai-sum, O., Chen, H. \& Chow, P.H. (2006). Male genital tract antioxidant enzymes - Their ability to preserve sperm DNA integrity. Mol. Cell. Endocrinol. 250, 80-83.

Wylde, Z., Spagopoulou, F., Hooper, A.K., Maklakov, A.A. \& Bonduriansky, R. (2019). Parental breeding age effects on descendants' longevity interact over 2 generations in matrilines and patrilines. PLoS Biol. 17, e3000556.

Xavier, M.J., Roman, S.D., Aitken, R.J. \& Nixon, B. (2019). Transgenerational inheritance: how impacts to the epigenetic and genetic information of parents affect offspring health. Hum. Reprod. Update 25, 519-541. 


\section{Xie, K., Ryan, D.P., Pearson, B.L., Henzel, K.S., Neff, F., Vidal, R.O. et al. (2018). Epigenetic alterations} in longevity regulators, reduced life span, and exacerbated aging-related pathology in old father offspring mice. Proc. Natl. Acad. Sci. 115, E2348-E2357.

Zhang, W., Qu, J., Liu, G.-H. \& Belmonte, J.C.I. (2020). The ageing epigenome and its rejuvenation. Nature Rev. Mol. Cell Biol. 21, 137-150.

Table 1. Linear mixed effects model exploring the effect of parental age on the number of sperm in the ejaculate (log-transformed). The model included offspring age (log-transformed), squared offspring age, maternal age, paternal age, the interactions between offspring age and parental age, and age at first and last insemination as fixed effects. Offspring, maternal and paternal identities, year of birth and year of data collection were included as random effects. The analysis was based on 11,107 observations collected over 17 years on 1,708 individuals (produced by 989 dams and 682 sires over 17 cohorts). We report parameter estimates (with SE and $95 \% \mathrm{Cl}$ ), $t$ and $p$ values for the initial model and the minimal adequate model.

\begin{tabular}{|c|c|c|c|c|c|}
\hline \multicolumn{6}{|l|}{ Initial model } \\
\hline Fixed effects & $\begin{array}{c}\text { Parameter } \\
\text { estimate }\end{array}$ & $S E$ & $t$ & $p$ & $95 \% \mathrm{Cl}$ \\
\hline Intercept & 0.491 & 0.306 & & & \\
\hline Age at first insemination & -0.009 & 0.025 & -0.37 & 0.7109 & $-0.057 / 0.039$ \\
\hline Age at last insemination & 0.180 & 0.010 & 17.95 & $<0.0001$ & $0.161 / 0.200$ \\
\hline Offspring age & 1.538 & 0.146 & 10.52 & $<0.0001$ & $1.251 / 1.824$ \\
\hline Offspring age ${ }^{2}$ & -0.774 & 0.056 & -13.85 & $<0.0001$ & $-0.883 /-0.664$ \\
\hline Dam age & -0.001 & 0.022 & -0.03 & 0.9739 & $-0.044 / 0.043$ \\
\hline Sire age & -0.107 & 0.022 & -4.84 & $<0.0001$ & $-0.150 /-0.064$ \\
\hline Offspring age $x$ Dam age & -0.010 & 0.027 & -0.36 & 0.7209 & $-0.063 / 0.043$ \\
\hline Offspring age $x$ Sire age & 0.130 & 0.028 & 4.73 & $<0.0001$ & $0.076 / 0.184$ \\
\hline Offspring age ${ }^{2} \times$ Dam age & 0.005 & 0.009 & 0.48 & 0.6306 & $-0.014 / 0.023$ \\
\hline Offspring age ${ }^{2} x$ Sire age & -0.038 & 0.010 & -3.97 & $<0.0001$ & $-0.057 /-0.019$ \\
\hline Random effects & Variance & $S E$ & $z$ & $p$ & \\
\hline Individual ID & 0.574 & 0.038 & 14.91 & $<0.0001$ & \\
\hline Dam ID & 0.107 & 0.031 & 3.41 & 0.0003 & \\
\hline Sire ID & 0.030 & 0.020 & 1.47 & 0.0713 & \\
\hline Year of birth & 0.014 & 0.010 & 1.42 & 0.0778 & \\
\hline Year of data collection & 1.177 & 0.444 & 2.65 & 0.0040 & \\
\hline Residual & 0.521 & 0.008 & 67.76 & $<0.0001$ & \\
\hline
\end{tabular}

Minimal adequate model

\begin{tabular}{llllll}
\hline Fixed effects & $\begin{array}{c}\text { Parameter } \\
\text { estimate }\end{array}$ & $S E$ & $t$ & $p$ & $95 \% \mathrm{Cl}$ \\
\hline
\end{tabular}




\begin{tabular}{lccccc} 
Intercept & 0.489 & 0.295 & & & \\
Age at first insemination & -0.009 & 0.025 & -0.38 & 0.7006 & $-0.058 / 0.039$ \\
Age at last insemination & 0.180 & 0.010 & 17.95 & $<0.0001$ & $0.160 / 0.200$ \\
Offspring age & 1.509 & 0.113 & 13.36 & $<0.0001$ & $1.287 / 1.730$ \\
Offspring age $^{2}$ & -0.758 & 0.046 & -16.54 & $<0.0001$ & $-0.848 /-0.669$ \\
Sire age & -0.106 & 0.022 & -4.83 & $<0.0001$ & $-0.150 /-0.063$ \\
Offspring age x Sire age & 0.129 & 0.027 & 4.70 & $<0.0001$ & $0.075 / 0.183$ \\
Offspring age $^{2}$ x Sire age & -0.038 & 0.010 & -3.93 & $<0.0001$ & $-0.057 /-0.019$ \\
\hline Random effects & Variance & $S E$ & $z$ & $p$ & \\
\hline Individual ID & 0.573 & 0.038 & 14.93 & $<0.0001$ & \\
Dam ID & 0.107 & 0.031 & 3.42 & 0.0003 & \\
Sire ID & 0.030 & 0.020 & 1.46 & 0.0721 & \\
Year of birth & 0.014 & 0.010 & 1.42 & 0.0775 & \\
Year of data collection & 1.165 & 0.439 & 2.66 & 0.0039 & \\
Residual & 0.521 & 0.008 & 67.77 & $<0.0001$ & \\
\hline \hline
\end{tabular}


Table 2. Linear mixed effects models exploring the effect of patrilineal age (paternal, grandpaternal and great grandpaternal ages) on the number of sperm in the ejaculate (log-transformed). We ran 6 models contrasting patrilineal ages (yyy vs. oyy; yyy vs. yyo; yyy vs. yoo; yoo vs. oyy; yyo vs. yoo; yoo; oyy; the first letter referring to the paternal age, the second letter to the grandpaternal age, the third letter to great grandpaternal age). Offspring age (log-transformed), squared offspring age, patrilineal age, the interactions between offspring age and patrilineal age, age at first and last insemination were included as fixed effects. Offspring, maternal and paternal identities, year of birth and year of data collection were included as random effects. The analysis was based on 5,504 observations collected over 15 years on 888 males (produced by 624 dams and 394 sires over 16 cohorts). We report parameter estimates (with SE and $95 \% \mathrm{Cl}$ ), $t$ and $p$ values. Only the results of the fixed effects are reported.

\begin{tabular}{|c|c|c|c|c|c|}
\hline Fixed effects & $\begin{array}{c}\text { Parameter } \\
\text { estimate }\end{array}$ & $S E$ & $t$ & $p$ & $95 \% \mathrm{Cl}$ \\
\hline \multicolumn{6}{|l|}{ yyy vs. oyy } \\
\hline Intercept & 0.161 & 0.328 & & & \\
\hline Age at first insemination & -0.010 & 0.046 & -0.21 & 0.8324 & $-0.100 / 0.081$ \\
\hline Age at last insemination & 0.244 & 0.021 & 11.68 & $<0.0001$ & $0.203 / 0.285$ \\
\hline Offspring age & 1.729 & 0.099 & 17.42 & $<0.0001$ & $1.534 / 1.923$ \\
\hline Offspring age ${ }^{2}$ & -0.881 & 0.057 & -15.36 & $<0.0001$ & $-0.993 /-0.768$ \\
\hline Patrilineal age (oyy) & -0.949 & 0.210 & -4.52 & $<0.0001$ & $-1.360 /-0.538$ \\
\hline Offspring age $x$ patrilineal age (oyy) & 1.127 & 0.276 & 4.08 & $<0.0001$ & $0.586 / 1.669$ \\
\hline Offspring age ${ }^{2} x$ patrilineal age (oyy) & -0.287 & 0.103 & -2.79 & 0.0053 & $-0.489 /-0.085$ \\
\hline \multicolumn{6}{|l|}{ yyy vs. yyo } \\
\hline Intercept & 0.442 & 0.288 & & & \\
\hline Age at first insemination & -0.019 & 0.040 & -0.48 & 0.6288 & $-0.097 / 0.058$ \\
\hline Age at last insemination & 0.205 & 0.018 & 11.28 & $<0.0001$ & $0.169 / 0.241$ \\
\hline Offspring age & 1.679 & 0.095 & 17.75 & $<0.0001$ & $1.494 / 1.865$ \\
\hline Offspring age ${ }^{2}$ & -0.817 & 0.050 & -16.38 & $<0.0001$ & $-0.915 /-0.719$ \\
\hline Patrilineal age (yyo) & -0.434 & 0.140 & -3.11 & 0.0019 & $-0.708 /-0.161$ \\
\hline Offspring age $x$ patrilineal age (yyo) & 0.484 & 0.160 & 3.02 & 0.0025 & $0.170 / 0.798$ \\
\hline Offspring age ${ }^{2} x$ patrilineal age (yyo) & -0.120 & 0.055 & -2.18 & 0.0292 & $-0.228 /-0.012$ \\
\hline \multicolumn{6}{|l|}{ yyy vs. yoo } \\
\hline Intercept & 0.305 & 0.311 & & & \\
\hline Age at first insemination & -0.01 & 0.047 & -0.20 & 0.8390 & $-0.102 / 0.083$ \\
\hline Age at last insemination & 0.224 & 0.021 & 10.84 & $<0.0001$ & $0.184 / 0.265$ \\
\hline Offspring age & 1.671 & 0.096 & 17.39 & $<0.0001$ & $1.483 / 1.859$ \\
\hline Offspring age ${ }^{2}$ & -0.827 & 0.055 & -15.00 & $<0.0001$ & $-0.935 /-0.719$ \\
\hline Patrilineal age (yoo) & 0.890 & 0.293 & 3.04 & 0.0024 & $0.315 / 1.465$ \\
\hline Offspring age $x$ patrilineal age (yoo) & -0.901 & 0.356 & -2.53 & 0.0113 & $-1.599 /-0.204$ \\
\hline Offspring age $\mathrm{e}^{2}$ patrilineal age (yoo) & 0.278 & 0.132 & 2.10 & 0.0356 & $0.019 / 0.538$ \\
\hline
\end{tabular}




\begin{tabular}{|c|c|c|c|c|c|}
\hline \multicolumn{6}{|l|}{ yyo vs. oyy } \\
\hline Intercept & 0.719 & 0.331 & & & \\
\hline Age at first insemination & -0.049 & 0.060 & -0.82 & 0.4135 & $-0.166 / 0.068$ \\
\hline Age at last insemination & 0.119 & 0.030 & 3.93 & $<0.0001$ & $0.059 / 0.178$ \\
\hline Offspring age & 2.214 & 0.158 & 14.03 & $<0.0001$ & $1.904 / 2.523$ \\
\hline Offspring age ${ }^{2}$ & -0.667 & 0.063 & -10.57 & $<0.0001$ & $-0.791 /-0.544$ \\
\hline Patrilineal age (oyy) & -0.679 & 0.240 & -2.83 & 0.0047 & $-1.150 /-0.209$ \\
\hline Offspring age $x$ patrilineal age (oyy) & 0.736 & 0.302 & 2.44 & 0.0147 & $0.145 / 1.328$ \\
\hline Offspring age ${ }^{2} \times$ patrilineal age (oyy) & -0.211 & 0.110 & -1.91 & 0.0566 & $-0.427 / 0.006$ \\
\hline \multicolumn{6}{|l|}{ yyo vs. yoo } \\
\hline Intercept & 1.329 & 0.241 & & & \\
\hline Age at first insemination & -0.090 & 0.057 & -1.59 & 0.1130 & $-0.201 / 0.021$ \\
\hline Age at last insemination & 0.028 & 0.022 & 1.29 & 0.1967 & $-0.015 / 0.071$ \\
\hline Offspring age & 2.139 & 0.148 & 14.43 & $<0.0001$ & $1.848 / 2.430$ \\
\hline Offspring age ${ }^{2}$ & -0.640 & 0.059 & -10.94 & $<0.0001$ & $-0.755 /-0.526$ \\
\hline Patrilineal age (yoo) & 1.317 & 0.298 & 4.42 & $<0.0001$ & $0.732 / 1.902$ \\
\hline Offspring age $x$ patrilineal age (yoo) & -1.286 & 0.367 & -3.51 & 0.0005 & $-2.006 /-0.567$ \\
\hline Offspring age ${ }^{2} x$ patrilineal age (yoo) & 0.337 & 0.135 & 2.49 & 0.0129 & $0.071 / 0.602$ \\
\hline \multicolumn{6}{|l|}{ yoo vs. oyy } \\
\hline Intercept & 1.667 & 0.514 & & & \\
\hline Age at first insemination & 0.005 & 0.104 & 0.05 & 0.9596 & $-0.200 / 0.210$ \\
\hline Age at last insemination & 0.134 & 0.051 & 2.63 & 0.0087 & $0.034 / 0.235$ \\
\hline Offspring age & 1.473 & 0.405 & 3.64 & 0.0003 & $0.678 / 2.269$ \\
\hline Offspring age ${ }^{2}$ & -0.581 & 0.165 & -3.52 & 0.0005 & $-0.905 /-0.257$ \\
\hline Patrilineal age (oyy) & -1.859 & 0.361 & -5.16 & $<0.0001$ & $-2.567 /-1.151$ \\
\hline Offspring age $x$ patrilineal age (oyy) & 1.760 & 0.454 & 3.88 & 0.0001 & $0.868 / 2.652$ \\
\hline Offspring age ${ }^{2} \times$ Patrilineal age (oyy) & -0.443 & 0.170 & -2.61 & 0.0093 & $-0.776 /-0.110$ \\
\hline
\end{tabular}


529 Figure 1. Age-dependent trajectories of offspring sperm number in the ejaculate (log-transformed

$530 \times 10^{6}$ ) as a function of paternal age. A) We report the raw data (blue boxes $=1-5$ years old; red boxes

531 = over 5 years old) and B) the LMM predictions. Data on the $\mathrm{x}$-axis were slightly jittered to improve 532 readability.

533

534 Figure 2. A) Least-square means ( \pm SE) of number of sperm in the ejaculate (log-transformed $\left.\times 10^{6}\right)$ at 535 the age of 1 year, for the two paternal age classes. B) Least-square means ( $\pm \mathrm{SE}$ ) of number of sperm 536 in the ejaculate (log-transformed $\times 10^{6}$ ) over the whole life, for the two paternal age classes.

537

538 
Figure 1.

A

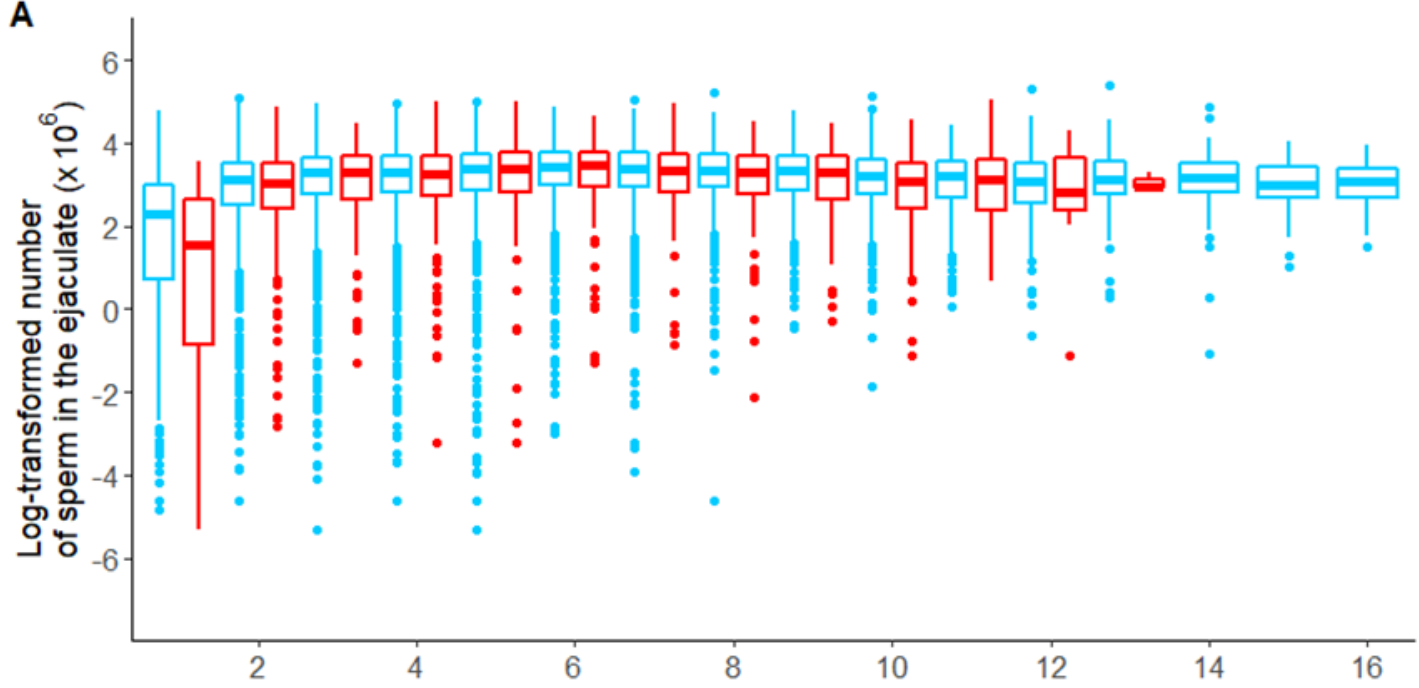

B

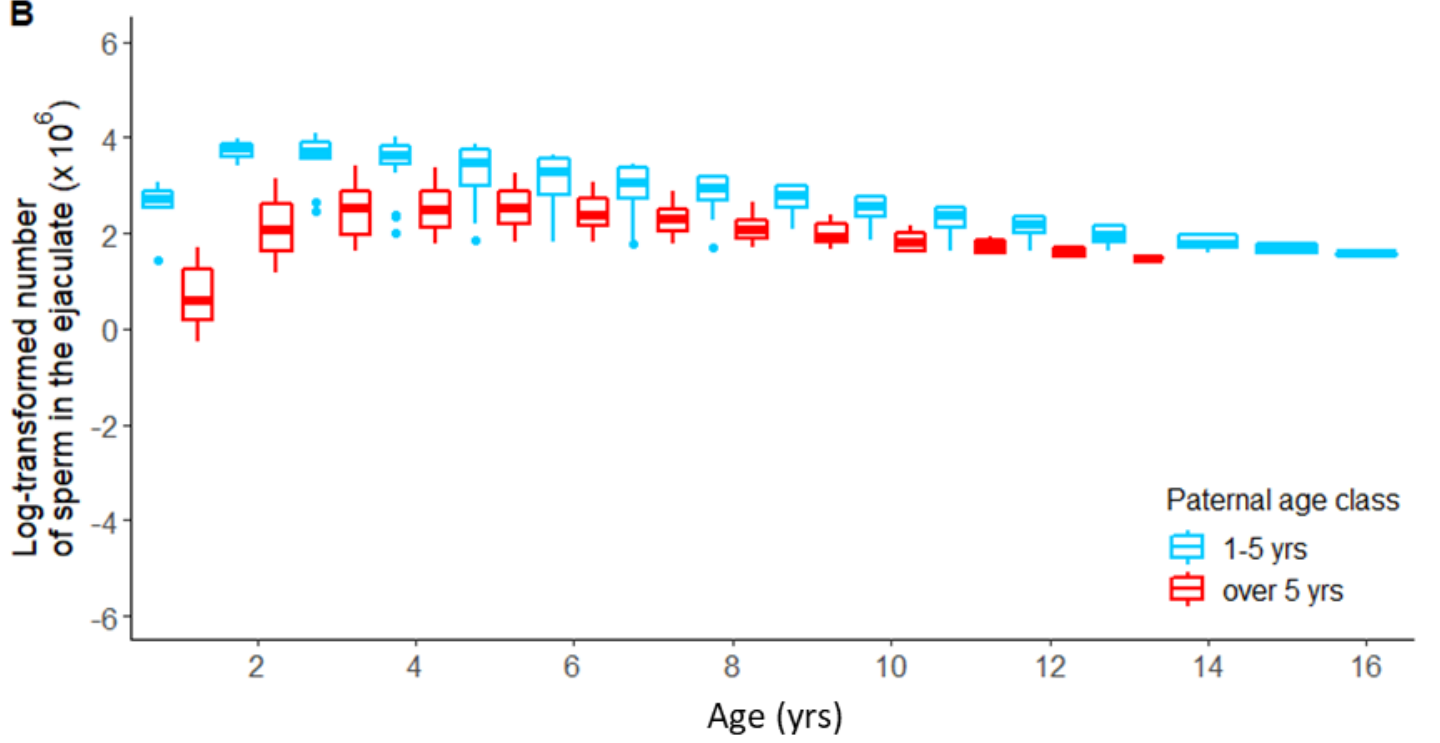

540

541

542

543

544

545

546 
Figure 2.

548

549
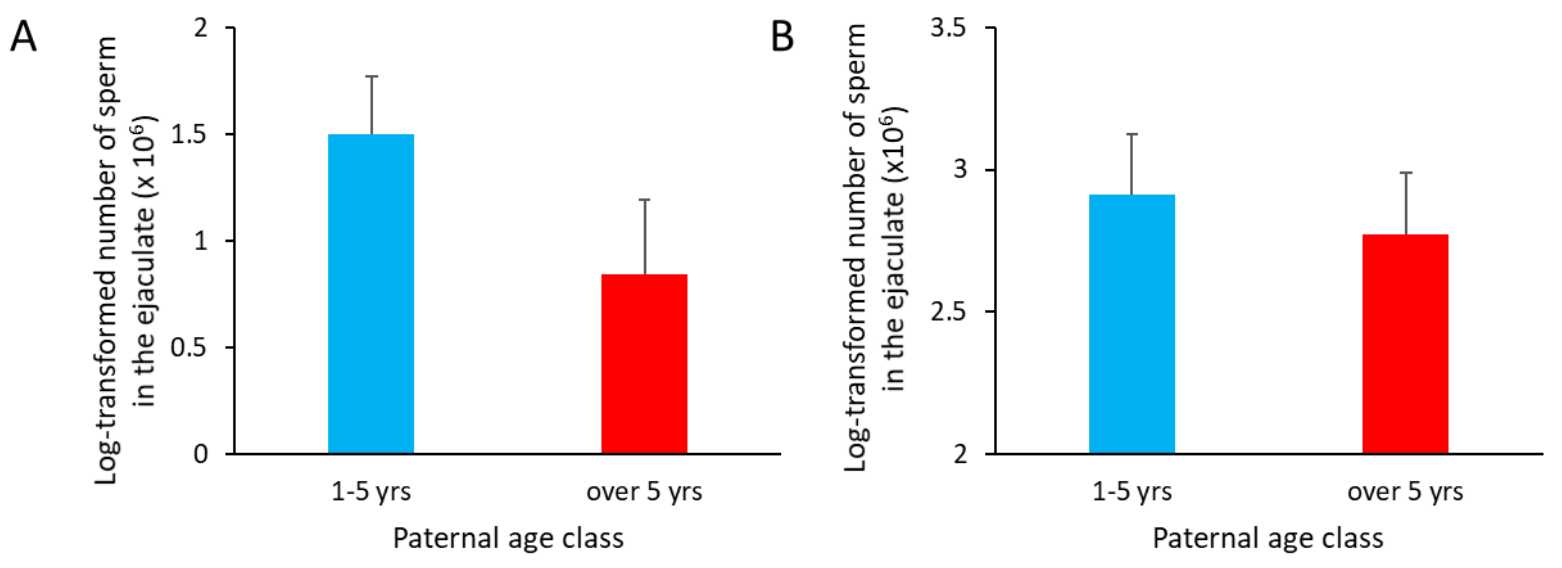

550

551

552

553

554 